\title{
Erratum
}

Somnologie $2020 \cdot 24: 115$

https://doi.org/10.1007/s11818-020-00244-x

Online publiziert: 28 . Februar 2020

(c) Springer Medizin Verlag GmbH, ein Teil von Springer Nature 2020

Kai Spiegelhalder ${ }^{1}$ Jens Acker ${ }^{2} \cdot$ Harald Baumeister $^{3} \cdot$ Antje Büttner-Teleaga $^{4,5}$. Heidi Danker-Hopfe ${ }^{6}$. David D. Ebert ${ }^{7} \cdot$ Ingo Fietze $^{8}$. Lukas Frase $^{1}$. Sarah Klein ${ }^{9}$. Dirk Lehr ${ }^{10} \cdot$ Andy Maun $^{11} \cdot$ Isabella Mertel ${ }^{12} \cdot$ Kneginja Richter $^{13} \cdot$ Dieter Riemann ${ }^{1}$. Cornelia Sauter ${ }^{6}$. Claudia Schilling ${ }^{14} \cdot$ Angelika A. Schlarb $^{15} \cdot$ Markus Specht $^{16}$. Lisa Steinmetz ${ }^{1} \cdot$ Hans G. Wee $\beta^{17} \cdot$ Tatjana Crönlein ${ }^{18}$

${ }^{1}$ Klinik für Psychiatrie und Psychotherapie, Universitätsklinikum Freiburg, Medizinische Fakultät, AlbertLudwigs-Universität Freiburg, Freiburg, Deutschland; ${ }^{2}$ Klinik für Schlafmedizin, Bad Zurzach, Schweiz; ${ }^{3}$ Abteilung für Klinische Psychologie und Psychotherapie, Universität Ulm, Ulm, Deutschland; ${ }^{4}$ Institut für Kognitive Wissenschaft, Woosuk Universität, Wanjugun, Südkorea; ${ }^{5}$ Fachbereich für Psychiatrie, Universität Witten/Herdecke, Witten, Deutschland; ${ }^{6}$ Kompetenzzentrum Schlafmedizin, Charité Universitätsmedizin Berlin, Berlin, Deutschland; ' ${ }^{7}$ Department of Clinical, Neuro- and Developmental Psychology, Vrije University Amsterdam, Amsterdam, Niederlande; ${ }^{8}$ Interdisziplinäres Schlafmedizinisches Zentrum, Charité - Universitätsmedizin Berlin, Berlin, Deutschland; ' ${ }^{9}$ BARMER, Schwäbisch-Gmünd, Deutschland; ${ }^{10}$ Abteilung für Gesundheitspsychologie, Leuphana Universität Lüneburg, Lüneburg, Deutschland; " Lehrbereich Allgemeinmedizin, Medizinische Fakultät, Universitätsklinikum Freiburg, Freiburg, Deutschland; ${ }^{12}$ Schön Klinik Roseneck, Prien am Chiemsee, Deutschland; ${ }^{13}$ Klinik für Psychiatrie und Psychotherapie, Klinikum Nürnberg Nord, Nürnberg, Deutschland; ${ }^{14}$ Zentralinstitut für Seelische Gesundheit, Klinik für Psychiatrie und Psychotherapie, Medizinische Fakultät Mannheim, Universität Heidelberg, Mannheim, Deutschland; ${ }^{15}$ Fakultät für Psychologie und Sportwissenschaft, Arbeitseinheit Klinische Psychologie und Psychotherapie des Kindesund Jugendalters, Universität Bielefeld, Bielefeld, Deutschland; ${ }^{16}$ Zentrum für interdisziplinäre Schlafmedizin, DKD HELIOS Klinik Wiesbaden, Wiesbaden, Deutschland; ${ }^{17}$ Schlafzentrum am Pfalzklinikum Klingenmünster, Klingenmünster, Deutschland; ${ }^{18}$ Klinik für Psychiatrie und Psychotherapie, Universität Regensburg, Regensburg, Deutschland

\section{Erratum zu: Digitale Behandlungsangebote für Insomnie - eine Übersichtsarbeit}

\section{Erratum zu:}

Somnologie 2020

https://doi.org/10.1007/s11818-020-

00238-9

In diesem Beitrag hat sich unter „Einhaltung ethischer Richtlinien“ ein Fehler bei der Angabe der Interessenkonflikte eingeschlichen.

Korrekt ist: David D. Ebert und Dirk Lehr sind Gesellschafter des GET.ON Instituts.

Die fehlende Nennung dieses Interessenkonflikts wurde durch einen Bearbeitungsfehler des Erstautors des Artikels bedingt und nicht durch die beiden genannten Autoren.

Die korrekte Angabe der Interessenkonflikte lautet wie folgt:

Interessenkonflikt K. Spiegelhalder, J. Acker, H. Baumeister, A. BüttnerTeleaga, H. Danker-Hopfe, I. Fietze,
L. Frase, A. Maun, I. Mertel, K. Richter, D. Riemann, C. Sauter, C. Schilling, A. A. Schlarb, M. Specht, L. Steinmetz, H. G. Weeß und T. Crönlein geben an, dass kein Interessenkonflikt besteht. S. Klein ist Mitarbeiterin der BARMER. D. D. Ebert und D. Lehr sind Gesellschafter des GET.ON Instituts.

\section{Korrespondenzadresse}

Prof. Dr. Dr. Kai Spiegelhalder

Klinik für Psychiatrie und Psychotherapie, Universitätsklinikum Freiburg, Medizinische Fakultät, Albert-Ludwigs-Universität Freiburg Hauptstr. 5, 79104 Freiburg, Deutschland kai.spiegelhalder@uniklinik-freiburg.de

Die Online-Version des Originalartikels ist unter https://doi.org/10.1007/s11818-020-00238-9 zu finden. 